Enfoques. Revista de Investigación en Ciencias de la Administración http://doi.org/10.33996/revistaenfoques.v4i13.76 No. 13 | Volumen 4 | Enero - Marzo 2020 http://revistaenfoques.org ISSN: $2016-8219$

\section{MODELO DE EJECUCIÓN DE PROYECTOS DE VIVIENDAS DE INTERÉS SOCIAL DEL SECTOR CONSTRUCCIÓN}

\author{
MODEL OF EXECUTION OF SOCIAL INTEREST HOUSING \\ PROJECTS OF THE CONSTRUCTION SECTOR
}

\author{
Elizabeth Dubuc
}

Artículo recibido octubre 2019 | Arbitrado noviembre 2019 | Publicado 01 de enero 2020

\section{Resumen}

La presente investigación tuvo como objetivo proponer un modelo gerencial para la fase estado Zulia, sustentándose teóricamente en los aportes de Cartay, Pmbok, y Chamoun, entre otros. La metodología fue de tipo descriptiva, con diseño transeccional de campo. La técnica de recolección de la información utilizada fue la encuesta con un instrumento cuestionario contentivo de 65 ítems. Se identificaron las fases de un modelo gerencial para la fase de ejecución de proyectos de viviendas de interés social del sector construcción del Edo Zulia, los cuales quedan plenamente reseñados en la propuesta del modelo. Los resultados evidenciaron debilidades en las fase de ejecución por lo que se considera que las empresas aplicar un nuevo modelo de gerencia en la dirección de proyectos civiles en su fase de ejecución, con la finalidad de optimar el seguimiento y control del proyecto civil y su rentabilidad.

Palabras clave: Modelo gerencial; fase de ejecución; viviendas de interés social

\begin{abstract}
The objective of this research was to propose a management model for the execution phase of social interest housing projects in the construction sector of Zulia State, theoretically based on the contributions of Cartay, Pmbok, and Chamoun, among others. The methodology was descriptive, with transectional field design. The information collection technique used was the survey with a 65 -item content questionnaire instrument. The phases of a management model were identified for the execution phase of social interest housing projects in the construction sector of Edo Zulia, which are fully outlined in the model proposal. The results showed weaknesses in the execution phase, so it is considered that companies apply a new management model in the direction of civil projects in their execution phase, in order to optimize the monitoring and control of the civil project and its profitability.
\end{abstract}

Key words: Management model; execution phase; social interest housing

\section{Elizabeth Dubuc}

dubucelizabeth62@gmail.com Universidad Rafael Belloso Chacín, Venezuela.

Ingeniero Civil, Magister en Gerencia de Proyecto Industriales de la Universidad Rafael Belloso Chacín 


\section{INTRODUCCIÓN}

En el mundo cambiante de hoy las empresas y organizaciones tanto públicas como privadas, se mantienen en constante competencia en los mercados, por ende la efectividad y los logros de los objetivos planteados dependen en gran manera de la habilidad y/o capacidad de gestión que posea el gerente o líder de proyecto para el manejo de sus proyectos al igual que de la cooperación que obtenga de su equipo de trabajo, cabe destacar como todo procedimiento de gestión se interconecta y depende de la mencionada habilidad de gestionar y planificar las actividades y de ello depende al mismo tiempo el éxito del proyecto que se quiera desarrollar.

Cuando se habla de habilidades y condiciones para gestionar y planificar actividades que aseguren el éxito del proyecto, ciertamente están éstas referidas a aquellas capacidades innatas o aprendidas del individuo para el manejo eficiente de diferentes circunstancias propias de la actividad administrativa, en otras palabras, se refiere a la capacidad y/o habilidad para gerenciar una actividad, en este caso específico un proyecto.

En efecto, Chamoun, (2007), define gerencia como la aplicación de conocimientos habilidades, técnicas y herramientas a las actividades del proyecto con el fin de superar las expectativas de los involucrados.

De igual manera, Krygier (2010), la define como un cuerpo de conocimientos aplicables a la dirección efectiva de una organización. En la actualidad existe un consenso, entre muchos autores, al señalar que el término gerencia se puede definir como un proceso que implica la coordinación de todos los recursos disponibles en una organización: Humanos, físicos, tecnológicos, financieros.
De esta manera, se pueden distinguir tres (3) aspectos claves de definir la gerencia como proceso:

- En primer lugar, la coordinación de recursos de la organización.

- En Segundo, la ejecución de funciones gerenciales 0 también llamadas administrativas como medio de lograr la coordinación.

- En tercer lugar, establecer el propósito del proceso gerencial, es decir, donde se quiere llegar o que es lo que se desea lograr.

- Determinar el propósito del proceso gerencial, se refiere, donde se quiere llegar o que es lo que se desea lograr. Al analizar las funciones gerenciales se encuentra respuesta al qué hace la gerencia.

Asimismo, Cartay (2010), define la gerencia de proyectos como la planificación, organización, dirección, y control de los recursos asignados a un proyecto que debe ser completado para alcanzar metas y objetivos específicos, expone que la gerencia de proyectos permite acometer tareas que no pueden ser manejadas eficientemente mediante las estructuras organizativas tradicionales, por lo que tiene que ser considerada como una plataforma fundamental en la búsqueda de nuevas formas de organización futura, diseñadas para integrar esfuerzos complejos $y$ disminuir la burocracia. Igualmente, la gerencia de proyectos permite acometer la ejecución de actividades con un mínimo de interrupción para el funcionamiento normal de los negocios.

De acuerdo a estas definiciones, la gerencia es un tipo de administración cuyo rasgo particular es el énfasis en la búsqueda 
de la eficiencia, es decir, los procesos administrativos deben transformarse, tomar características que permitan no solo obtener los fines, sino resultados económicos favorables.

Es así como un gerente debe tener la capacidad de manejar no solo conocimiento, técnico, y equipo humano, sino que también se espera de él que tenga habilidades y destrezas, liderazgo, comunicación efectiva, capacidad para hacer que las cosas sucedan, brindar soluciones, saber adaptarse a los cambios constantes e integrar el equipo de trabajo, saber implementar procesos de gestión administrativas que garanticen el éxito y rentabilidad del proyecto, así como el éxito de la gestión.

En el caso específico de esta investigación, en las empresas contratistas del sector de la construcción se desarrollan proyectos que presentan desfases en sus diferentes etapas los cuales impactan la fase de ejecución ya que se generan retrasos en la culminación de la obra, lo que origina cambios de alcance, y esto a su vez ocasiona demoras que afectan negativamente el presupuesto original y el tiempo programado de entrega de la obra.

Efectivamente, de la problemática expuesta nace el objetivo de esta investigación, como lo es proponer un modelo gerencial para la fase de ejecución de proyectos de viviendas de interés social del sector construcción del estado Zulia.

\section{Fundamentación teórica}

Conocer las características del proyecto es uno de los principales aspectos a considerar cuando se implementan nuevas maneras de gerenciar y en especial durante la fase de ejecución de los proyectos, etapa cuando se deben llevar a cabo todas las actividades planificadas. Toda empresa antes de iniciar un proyecto debe conocer en detalle, lo que va a ejecutar; para lo cual es necesario tener a mano las especificaciones, planos, normas, entre otros y mantener comunicación constante con el ente contratante.

Entre los elementos que conforman las características del trabajo se encuentra: el tipo de construcción a ejecutar, el monto de inversión, tiempo de ejecución, finalidad del proyecto, y asignación presupuestaria.

En este sentido, Merrit, Loftin, Ricketts (2004), mencionan que es necesario hacer una descripción de manera concisa de la naturaleza y los alcances del trabajo en la sección correspondiente, así como de sus características inherentes, se incluye en ello los requisitos necesarios para que el trabajo se apegue a los planos y especificaciones.

Los autores enfatizan la necesidad de definir oportunamente, con claridad y bien detallado, todo lo relacionado con el tipo de construcción, ya que esta será una guía para dar inicio al trabajo.

En cuanto al monto de inversión, Campero, (2007), menciona que la inversión que compromete la fase de ejecución fluctúa entre el $85 \%$ y el $95 \%$ del presupuesto del proyecto, por lo que resulta mu

Según, Merrit, Loftin, Ricketts (2004), para operar de manera eficiente, una empresa debe estar capacitada para evaluar y analizar su estado financiero en todo momento, así como de compilar y registrar todas las transacciones relativas al aspecto financiero.

Según, Córdoba (2006), en el marco financiero se especifican las necesidades de recursos a invertir, con detalles de las cantidades y fechas para los diversos ítems señalados, su forma de financiación y las estimaciones de ingresos y egresos para el periodo de vida útil del proyecto, lo que 
permite establecer los recursos que demanda el proyecto, los egresos e ingresos que generarían y la manera como se financiará.

En efecto, se puede decir que el monto de inversión es el recurso necesario para saber si es viable o no el proyecto y, de serlo gerenciar de manera óptima el contrato para conseguir rentabilidad económica del mismo. El enfoque de Campero (2007), se dirige a los recursos financieros, antes durante y culminación del proyecto, en su fase de ejecución; mientras que Córdoba, (2006) y Merrit, Loftin y Ricketts (2004), se refieren al monto de inversión, como los recursos necesarios antes, durante y después de la culminación del proyecto.

En relación con el tiempo de ejecución, Cartay (2010), expresa que la gestión del tiempo está constituida por los procesos necesarios para garantizar la finalización oportuna (a tiempo) del proyecto, sus documentos o productos entregables comunes son: cronograma de trabajo, calendario de ejecución, estimación de la duración de las actividades programas del proyecto.

Según, PMBOK (2008), tiempo de ejecución se refiere a los procesos necesarios para lograr la conclusión del proyecto a tiempo. Los procesos de gestión de tiempo del proyecto incluyen la definición de las actividades, el establecimiento de la secuencia de las actividades, la estimación de recursos de las actividades, la estimación de la duración de actividades y el control del cronograma.

Estos procesos interaccionan entre si y también con los procesos de las demás áreas de conocimiento. Cada proceso puede implicar el esfuerzo de una o más personas, dependiendo de las necesidades del proyecto. Cada proceso tiene lugar por lo menos una vez en cada proyecto y se produce en una o más fases, si el proyecto se encuentra divido en fase.

De igual manera el PMBOK se refiere al tiempo de ejecución como los tiempos estipulados para la entrega de la obra u proyecto, así como el tiempo de la ejecución de las actividades planificadas en el cronograma de trabajo.

Los autores sólo difieren en que el PMBOK (2008), describe el tiempo de ejecución de manera muchas más detallada y señala y al mismo tiempo explica de manera muy específica cada proceso que incluye la gestión del tiempo y sus componentes relacionados entre sí; mientras que el primer autor, Cartay, se refiere el tiempo de ejecución, como el objetivo de cumplir los tiempos indicados en los programas de trabajo, para que de presentarse alguna desviación se pueda inmediatamente tomar acciones correctivas de la misma, sin generar así retrasos en el plan de trabajo general del proyecto.

En este mismo orden de ideas, Cartay (2010), expresa que la finalidad del proyecto tiene que ver con su "por qué" y su alineamiento con el plan de negocios. Es conocer las metas y los objetivos, los cuales expresan los resultados esperados del proyecto y "qué" se satisface con los mismos.

Asimismo, Campero (2007), expresa que la finalidad del proyecto tiene que ver con la función que debe cumplir el proyecto, con la producción esperada, tanto en volumen como en calidad de producto; e incluye también todas las acciones de la empresa que deberán tener como finalidad alcanzar eficazmente esas metas.

Según, Merrit, Loftin, Ricketts (2004), con las metas conocidas, el diseñador define 
los objetivos del sistema. Estos son similares a las metas, pero proporcionan en detalle los requerimientos que el sistema debe satisfacer para alcanzarlas.

Algunos objetivos, pueden ser la minimización de los costos iníciales, los costos del ciclo de vida o el tiempo de construcción, otros objetivos, que se aplican en el diseño de casi todos los proyectos son la salud, seguridad y bienestar que se mencionan en los reglamentos de la construcción. Los objetivos deben ser lo suficientemente específicos para guiar la planificación del proyecto y la selección de los componentes con características determinadas.

De los autores anteriormente mencionados, se puede definir finalidad del proyecto, como el "por qué" y "para qué" del proyecto, cuáles son sus metas y los beneficios a considerar.

De igual forma, Cartay (2010), refiere que la asignación presupuestaria está constituida por los procesos necesarios para garantizar que el proyecto se ciña al presupuesto acordado. Sus documentos o productos entregable comunes son: planificación de recursos, estimación de costos, presupuestos del proyecto.

Según, PMBOK (2008), la asignación presupuestaria incluye los procesos involucrados en la planificación, estimación, preparación del presupuesto y control de costos de forma que el proyecto se pueda completar dentro del presupuesto aprobado. Los procesos relacionados con esta área son: estimación de costos, preparación del presupuesto de costo, control de costos. La gestión de costos del proyecto se ocupa, principalmente del costo de los recursos necesarios para completar las actividades del cronograma.
La asignación de presupuesto, se refiere a controlar los costos asignados al proyecto, el líder o gerente de proyecto debe tener la competencia de gestionar de manera inteligente cuanto se ha ejecutado vs lo planificado.

A pesar de que los dos autores presentan conceptos similares, difieren ya que Cartay (2010), no detalla los procesos de gestión de costo como los describe el PMBOK (2008), donde la exposición del PMBOK considera la asignación presupuestaria, como la herramienta gerencial que todo líder o gerente debe aplicar para poder culminar el proyecto con los montos asignados, así como para evitar pérdidas financieras, por los inconvenientes que generalmente se presenta en el desarrollo del proyecto.

\section{MÉTODO}

La siguiente investigación fue de tipo proyectiva, por consistir en la elaboración de una propuesta, que nace de una necesidad planteada en las actividades reales de la ejecución de proyecto. Se considera de diseño transeccional, de campo, debido a que la recolección de información fue directamente en las empresas dedicada a la construcción que fueron seleccionadas como objeto de estudio, sin manipulación alguna de la variable en estudio.

La población de estudio de esta investigación estuvo conformada por 15 sujetos, que pertenecen al departamento de proyecto e inspección de las empresas contratistas, Coperca C.A, R\&S Construcciones, Inprolin C.A, encargadas de la fase de ejecución de viviendas de interés social en el estado Zulia. Debido a que la población de esta investigación es finita y accesible, se consideró como un censo poblacional. 
La técnica de recolección de información fue la encuesta y como instrumento se utilizó un cuestionario conformado por un total de 65 preguntas con 4 alternativas de respuesta, en la escala tipo Lickert. La validez del instrumento se efectuó mediante consulta a cincos expertos en el área de Gerencia de Proyectos y Metodología de la Investigación. En cuanto a la confiabilidad del instrumento se aplicó una prueba piloto a ocho sujetos (8) con similares características a la población determinada en dicho estudio, y luego se aplicó el coeficiente alpha cronbach, obteniéndose un resultado de 0,975 que muestra la alta confiabilidad del instrumento.

\section{RESULTADOS}

Los resultados de la aplicación del instrumento a la población estudiada arrojo existen desviaciones en el proceso de ejecución de los proyectos, afirmación que se hace por cuanto la medición de los procesos de gestión dieron resultado una mediana de 2,23 que al ser comparada con el baremo de interpretación indica que existe una baja gestión en la ejecución de los proyectos de construcción, lo cual induce a inferir que no hay una clara definición del alcance de los proyectos, lo cual trae como consecuencia que los recursos referidos a aspectos técnicos, económicos y humanos tienen grandes problemas de disponibilidad por cuanto la programación a partir de la definición del alcance presenta fallas.

De esta manera, se planteó la necesidad de proponer un modelo gerencial para la gestión de la ejecución de los proyectos de construcción.

\section{Modelo gerencial para la fase de ejecución de proyectos de viviendas de interés social del estado Zulia}

Para la creación de las propuestas se tomaron en cuenta las debilidades identificadas como situación problema en la fase de ejecución de los proyectos de construcción de viviendas de interés social en el estado Zulia, a fin de que la implementación de este modelo gerencial para la fase de ejecución de los proyectos, ayude a corregir en las empresas privadas y públicas de la construcción de viviendas de interés social en Venezuela, el cumplimiento oportuno de los aspectos administrativos planificados en los cronogramas de trabajo y por ende conlleve al éxito de los proyectos.

\section{Conceptualización}

El presente modelo de gerencia, consiste en un conjunto de herramientas que el gerente y su equipo de trabajo podrán implementar en su gestión de administración de proyecto durante la fase de ejecución, para contrarrestar efectos negativos tales como pérdidas económicas incumplimiento de los tiempo establecidos, la entrega de la procura a tiempo, el control del costo del proyecto, su planificación y ejecución dentro de un plan o cronograma de trabajo.

\section{Objetivos de la propuesta}

- Proporcionar a la gerencia un modelo para ser utilizado en la fase de ejecución, que permita a los gerentes, optimar los procesos del área administrativa del proyecto. 
- Mejorar el desempeño en la fase de ejecución de las gestiones administrativas de las empresas constructoras encargadas de proyectos de viviendas de interés social.

- Mejorar y aplicar la gestión de alcance de trabajo. Definición de alcance

- Optimar la gestión de tiempo, para que se cumplan las actividades en los tiempos previstos.

- Mejorar la gestión de costos y estimaciones del proyecto.

- Optimar la gestión de calidad (verificación de materiales y productos)

- Optimar la programación de actividades, creando cronogramas de trabajo o plan maestro, con ayuda de programas o software de control de obra.

- Mejorar el seguimiento de control de la obra.

- Mejorar los cierres de proyecto creando expedientes donde queden plasmados todos los documentos referentes al mismo.

Se persigue que los responsables del proyecto aprendan y se motiven para manejar e implementar herramientas gerenciales de manera efectiva, dándoles la debida importancia de su uso durante todas las fases del proyecto, pero con mayor énfasis durante la fase de ejecución, dejando así atrás los métodos tradicionales e informales, que conllevan pérdidas económicas del proyecto, así como de competitividad en el mercado de la construcción.

\section{Alcance}

Esta propuesta de modelo gerencial para la fase de ejecución de proyecto de viviendas de interés social del estado Zulia se podrá aplicar a todo tipo de empresa dedicada a la construcción, ya sea ésta pública o privada, así como a organismos públicos que se encuentren relacionados con la ejecución y administración de soluciones habitacionales. De igual manera podrá ser aplicado por gerentes, líderes de proyectos, ingenieros, personal administrativo y por toda persona interesada en aprender herramientas gerenciales para la buena gestión de un proyecto en su fase de ejecución, donde una buena planificación y gestión es la base del manejo de proyectos en cualquiera de sus fases.

Propuesta del modelo gerencial para la fase de ejecución de proyectos de viviendas de interés social del estado Zulia

A continuación, se describen los parámetros y funciones administrativas que deben establecerse para obtener una gerencia eficiente y exitosa durante la fase de ejecución de proyectos de viviendas de interés social.

\section{Inicio}

Todo inicio de un proyecto cual sea su fase, se presenta con mucho entusiasmo y a la vez con algo de confusión, por ello la importancia de plantear objetivos, metas y la justificación del proyecto seguidamente se presentan algunas consideraciones a tomar en cuenta al inicio de un proyecto en su fase de ejecución:

a) Decidir que se va hacer.

b) Porqué se va a realizar el proyecto.

c) Cuánto tiempo establecido conlleva ejecutar el proyecto.

d) Realizar el diagrama organizacional del proyecto. 
e) Definir roles y asignaciones.

f) Se debe documentar el inicio del proyecto para formalizarlo.

g) Recopilar experiencias e información de proyectos anteriores.

h) Conocer las restricciones que limitan al equipo ejecutor o del proyecto.

\section{Alcance de trabajo o proyecto}

a) El gerente debe conocer amplia y oportunamente de los requisitos del proyecto entregados por el ente contratante, planos, especificaciones técnicas, forma de pago, tipo de contrato descripción de las actividades a realizar, límites o restricciones y riegos, entre otros.

b) El gerente debe tener conocimiento claro y preciso de los costos antes de ejecutar el proyecto, sobre todo su conocimiento debe incluir la creación del presupuesto base del proyecto.

c) El gerente debe definir metas y objetivos de proyecto en conjunto con su equipo de trabajo.

d) El gerente debe tener habilidades para lograr mantener el equilibrio entre alcance, costo y tiempo.

e) El gerente debe tener conocimiento y habilidad para guiar la selección del equipo humano, en función de mantener el equilibrio del desarrollo del proyecto, de igual forma debe cuidar que la comunicación entre la organización, el gerente, y el ente contratante sea eficiente.

f) Para lograr el mencionado equilibrio entre alcance, costo y tiempo el gerente debe identificar y tomar muy en cuenta los posibles riesgos; monitorearlos y corregirlos, bien sea en el proceso de contratación, del tipo de contrato, la forma de pago y/o las estimaciones de costos entre otros. g) El equipo de trabajo deberá estar preparado para responder a los cambios que puedan presentarse durante el desarrollo del proyecto, ya que muchas veces los cambios en la definición de actividades, antes de ejecutarlas, pueden ser inevitables.

h) El gerente debe ser asertivo, para saber controlar los conflictos que puedan presentarse durante su gestión.

\section{Programación de actividades}

La programación de actividades, es la segunda herramienta gerencial a implementar, pues con la ayuda de cronogramas de trabajo y usos de los diferentes programas de actividades que existen en el mercado, pueden los gerentes llevar un mayor control del proyecto.

a) El gerente debe definir las actividades prioritarias de una manera lógica y secuencial.

b) El gerente debe utilizar para crear el cronograma, programas de software de control de obras que se manejan en el mercado tales como Project, primavera, entre otros. Realizar este tipo de cronograma da una visual completa del proyecto, y permite evaluar y controlar los tiempos, costos y asignación de actividades.

c) La programación de actividades ayuda al gerente a identificar previamente los posibles riesgos del proyecto de manera que se pueden aplicar correcciones antes de su ejecución.

d) El gerente debe verificar con antelación la disponibilidad de recursos materiales, equipos, mano de obra y costos. 
La programación de actividades es una herramienta que tiene como beneficios el hecho de permitir una actualización constante del cronograma, mediante la cual se visualiza el proyecto y permite comparar cronogramas planificados vs ejecución.

\section{Procura}

El gerente debe realizar previamente un plan de trabajo maestro donde:

Especifique los materiales y tome en cuenta los tiempos de cada actividad, de igual manera debe prever la agilización de compras, así como la inspección de equipos y materiales.

a) Especifique los equipos y herramientas necesarias en el proyecto.

b) El cronograma de ejecución debe reflejar, las fechas en que los materiales y equipos serán incorporados al proyecto.

c) Clasificación de materiales y equipos.

d) Mantener los registros de proveedores.

e) Coordinar informes periódicos para la previsión y control de procura.

f) Se debe controlar y verificar, la calidad y la cantidad de materiales de construcción y equipos.

g) Se debe controlar y verificar, las estimaciones de costos en conjunto con lo presupuestado, presupuesto base asignado para la actividad de procura, así como los materiales y equipos.

\section{Manejo de contrato}

Todo gerente debe conocer, manejar y gestionar el contrato del proyecto desde su inicio hasta su finalización, incluyendo los documentos que lo conllevan y el tipo de contrato a manejar.
En primer lugar, el gerente debe conocer el tipo de contrato, ya sea de precios unitarios, suma global, administración directa, entre otros. Si el contrato es de precios unitarios se deben tener en cuenta las siguientes consideraciones:

a) Firma del documento principal o contrato.

b) Gestionar el trámite de fianza de anticipo otorgada por un instituto bancario o empresa de seguro.

c) Firma del acta de inicio de la ejecución de proyecto.

d) Conocer los montos y fechas y documentar las valuaciones entregadas al ente contratante. Estas deben tramitarse en un lapso entre una y otra no menor de quince días (15), ni mayor de sesenta (60) días calendarios.

e) El gerente debe estar al tanto de las fechas, y si se puede, gestionar trámites de presupuesto de obras extras, reconsideración de precios de mano de obra o material, presupuesto de disminución, presupuesto de aumento, por parte del ingeniero 0 funcionario del ente contratante. Si el proyecto así lo requiere.

f) El gerente debe estar en conocimiento de reportes o comunicaciones de asuntos tratados tanto de su equipo de trabajo del proyecto, como los del ente contratante.

g) El gerente debe tramitar y documentar el acta de terminación de obra, el líder del proyecto o encargado debe notificar al ente contratante con diez días (10) de anticipación la culminación de la obra.

h) Debe de igual modo, el gerente, solicitar por escrito el acta de 
aceptación provisional de obra dentro del plazo de sesenta días (60), contados a partir de la fecha del acta de terminación. Esta solicitud debe estar acompañada de las mediciones finales, cuadro de cierres, documentos técnicos como planos definitivos, garantías de equipos o mantenimiento adecuado.

i) El gerente debe gestionar y documentar, trámites de lapso de garantía de la obra, este documento determina el lapso de garantía necesario.

j) El gerente debe comprobar si la obra no presenta defectos en sus instalaciones, equipos y que los servicios funcionen correctamente.

k) El gerente debe gestionar y documentar, actas de recepción definitiva.

I) El gerente debe gestionar y solicitar por escrito, al ente contratante, la recepción definitiva de la obra, lo que según gaceta oficial debe ser dentro de los treinta (30) días calendarios luego de presentar la solicitud.

\section{Inspección de obra}

La actividad de inspección de obra, consiste en el seguimiento periódico del proyecto para verificar que se cumplan las actividades planificadas en el cronograma de trabajo, los tiempos, los costos y las especificaciones del proyecto. El gerente debe realizar visitas periódicas, para verificar y comprobar el avance de los cronogramas.

\section{Forma de pago}

El gerente o líder del proyecto debe implementar ciertos lineamientos para controlar y verificar el pago del proyecto. a) Primeramente, conocer el tipo de contrato, ya sea de precios unitario, suma global, administración directa, entre otros.

b) Realizar o tramitar la fianza de anticipo, si el contrato lo requiere, y tomando en cuenta, el monto entregado por el ente contratante, deducirá un porcentaje por cada valuación entregada.

c) Asegurarse de la elaboración de las valuaciones.

d) El gerente debe verificar que en cada valuación se refleje la cantidad de obra o servicio ejecutado.

e) El gerente debe verificar los tiempos establecidos de entrega. Estos deben tramitarse en un lapso no menor de quince (15) días, ni mayor de sesenta (60) días, y verificar conjuntamente los lapsos de tiempo que toma el pago a los proveedores.

f) El gerente debe verificar, en todo momento, que el avance físico de la obra sea mayor que el avance económico.

g) De igual manera, el gerente debe presentar, recibir, y verificar las facturas, así como documentar como garantía todos los trámites de pagos realizados y recibidos durante el proyecto.

\section{Control}

El control de un proyecto es continuo durante todo el proyecto hasta su finalización. El seguimiento y control permite obtener información, realizar observaciones y corregir cuando se visualizan desviaciones; el seguimiento y control es una herramienta que proporciona de manera efectiva al gerente y/o equipo de trabajo una forma 
de consolidar y resumir grandes bloques de actividades detalladas, tales como:

a) Comparar los cronogramas de trabajo de las actividades definidas, aplicando de manera periódica la comparación de la ejecución real vs la ejecución planificada.

b) Como herramienta de apoyo en juntas o reuniones con los ingenieros o encargados, por lo menos una vez por semana.

c) Elaboración de informes y reportes semanales como mensuales, que permitan analizar en frio la situación del proyecto.

d) El gerente del proyecto debe realizar visitas periódicas al sitio de la ejecución del proyecto para verificar los cronogramas e informes y poder concretar, afrontar las reuniones de trabajo.

e) El gerente del proyecto debe aplicar acciones correctivas para que cualquier control sea efectivo y eficaz.

f) Aplicar gestión de cambios, es decir, al comparar la ejecución real vs la ejecución planificada, se reflejan aquellas actividades con problemas y desviaciones que originan resultados no esperados, tan pronto ocurra este tipo de evento, tanto los gerentes como los encargados, deben tomar medidas para corregir y afrontar acciones y de esa manera evitar retrasos y pérdidas o incrementos de costos de manera desproporcionada.

g) Apoyarse en los presupuestos del proyecto, para conocer cuáles son los desembolsos en relación con los objetivos planteados.

h) Mantener los cronogramas de trabajo actualizados, de esa manera se podrá visualizar, identificar y aplicar las debidas correcciones.

i) Un buen proceso de control mide el desempeño de la obra.

j) El gerente debe verificar de manera oportuna las variaciones y corregirlas mediante la planificación o cronograma de trabajo.

k) El gerente debe verificar constantemente los métodos de control.

I) Después del seguimiento se autoriza la ejecución de la actividad

\section{Cierre de contrato}

Para la culminación de la obra, o cierre de contrato el gerente o líder de proyecto debe finiquitar el acuerdo legal acordado con el ente contratante y realizar o gestionar un expediente final que incluye una serie de documentos, el cual será garantía de la finalización del contrato.

Los documentos a considerar para el cierre del contrato son los siguientes:

a) Tramitar, con previa notificación al ente contratante, todo lo referente a liberación de fianzas, entrega de garantías de equipos y mantenimiento, acta de terminación de obra, solicitar el acta de recepción provisional, acta de lapso de garantía de la obra, acta de recepción definitiva.

b) Crear un archivo del proyecto, donde se documente el contrato original, presupuesto de la obra, estimaciones, cotizaciones, facturas de pago, lista de proveedores, cronogramas finales de trabajo, actualización de base de datos, análisis de precios unitarios, duración de las actividades, reportes de cambios, informes, valuaciones, lecciones aprendidas, planos definitivos. 
c) Realizar un reporte final, donde se resalte lo más relevante del proyecto en su fase de ejecución, este acompañarse por el presupuesto y el cronograma de trabajo final. d) Aplicar un pequeño cuestionario al cierre del proyecto, que sirva para evaluar el desempeño del equipo del proyecto y visualizar las lecciones aprendidas.

\section{Inicio}

Definición

de Alcance

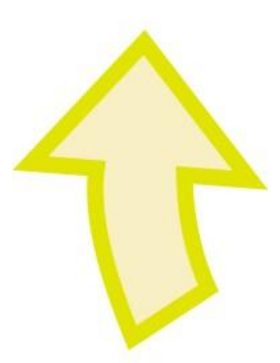

Cierre

Expedientefinal

Actas

Finanzas

Seguros

Valuaciones

Lecciones
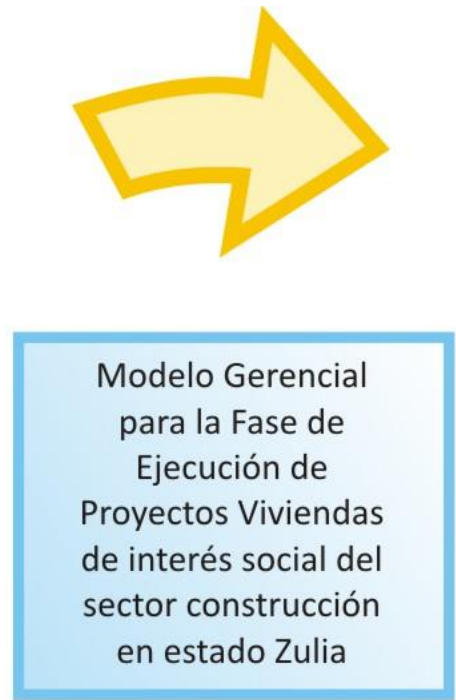

Zulia

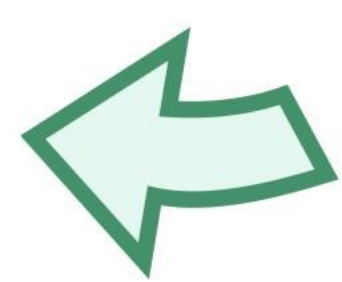

Planeación

Programación

de Actividades

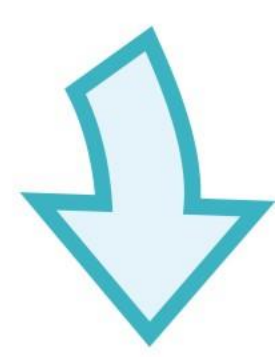

\section{Control}

Seguimiento,

Evaluación

Informes

Inspección

Figura 1. Modelo Gerencial para la fase de ejecución de proyectos de viviendas de interés social del Sector Construcción del estado Zulia.

\section{Factibilidad}

Para llevar a cabo esta propuesta es muy importante que el personal de las empresas, incluidos sus altos ejecutivos, tengan en cuenta que el objetivo de toda empresa, sea cual sea su naturaleza, es cumplir con la ejecución de sus proyectos en un tiempo determinado y con rentabilidad económica. De allí la importancia de implementar un modelo de gerencia efectivo en los proyectos civiles, y evitar así la administración subjetiva e informal, que genera serios problemas en el desarrollo de la obra.
Desde el punto de vista Humano, la propuesta es factible, por cuanto las empresas constructoras cuentan con el talento humano requerido, personal que está en capacidad y disposición de implementar este tipo de modelo y asesorar por medio de cursos de formación a quien así lo requiera.

Desde el punto de vista técnico, la propuesta de este modelo para optimar la gerencia de los proyectos, se puede implementar como un manual de procedimiento o guía que contempla y 
establece el procedimiento técnico, así como el desarrollo de la gestión de proyectos en su fase de ejecución.

Desde el punto de vista económico, la propuesta es factible ya que la disponibilidad de la inversión económica está garantizada por los dividendos que proveerá la aplicación de la propuesta.

El modelo que se presenta es una oportunidad de organizar y estructurar las actividades para optimar el desempeño de las empresas constructoras de viviendas de interés social durante la fase de ejecución para lograr así cubrir los objetivos planteados y dejar atrás la manera tradicional de gerenciar.

\section{CONCLUSIONES}

El modelo se elaboró como una herramienta administrativa para la planificación lógica y eficiente de las actividades que se presentan en la ejecución de proyecto de viviendas de interés social, a fin de manejar con éxito y minimizar los conflictos e imprevistos que se puedan presentarse en esta fase.

Igualmente, será una guía práctica en el uso de herramientas gerenciales y su implementación por parte de los líderes o gerentes de proyecto durante la fase de ejecución para proyectos civiles.

\section{REFERENCIAS}

Campero, M y Fernando, A (2007). Administración de Proyectos Civiles. 3 era Edición. Editorial. Universidad Católica de Chile. Chile

Cartay, I (2010). Planificación y control en la ejecución de Proyectos. Manual CIED. Maracaibo, Venezuela

Córdoba, M. (2006). Formulación y Evaluación de Proyectos. Editorial Eco Ediciones. Colombia

Chamoun, J. (2007). Administración Profesional de Proyectos - La Guía, Editorial McGraw-Hill Interamericana. Primera Edición México

Krygier, A (2010). Cultura Corporativa y Desarrollo. Editorial Krygier de Cohen Francis

Merrit, F., Loftin, M., y Kent, R. (2004). Manual Del Ingeniero Civil. 4ta edición. Editorial McGraw-Hill Companies, Inc., USA

PMBOK, (2008). Guía de los fundamentos para la dirección de proyectos.4ta. Edición. Editorial Project Management Institute, Inc. Pennsylvania, EEUU 